ISSN: 0210-7287

DOI: http://dx.doi.org/10.14201/161620199273283

\title{
LITERATURA COMPARADA E LITERATURA-MUNDO: ENQUADRAMENTO DISCIPLINAR ${ }^{1}$
}

\section{Comparative Literature and World Literature: A Disciplinary Framework}

\author{
Patrícia INFANTE DA CÂMARA \\ Universidade de Lisboa \\ anapatriciainfante@gmail.com
}

Recibido: junio de 2019; Aceptado: septiembre de 2019; Publicado: diciembre de 2019

Ref. Bibl. PATRÍCIA INFANTE DA CÂMARA. LITERATURA COMPARADA E LITERATURA-MUNDO: ENQUADRAMENTO DISCIPLINAR. 1616: Anuario de Literatura Comparada, 9 (2019), 273-283

RESUMO: Pese embora a recente criação de revistas científicas especializadas no campo (como o Journal of World Literature), a ocorrência de congressos e eventos culturais que lhe são dedicados (como o Festival de Literatura-Mundo do Sal), a criação de instituições que reúnem e representam os seus praticantes (como o Institute for World Literature, na Universidade de Harvard) e até mesmo a sua inserção no âmbito dos curricula universitários (sobretudo os norte-americanos, onde não obstante assumem tendencialmente a configuração de survey courses consagrados apenas à listagem e breve apresentação de textos literários "do mundo"), creio que seja no âmbito da Literatura Comparada que a literatura-

1. Este artigo insere-se no âmbito de uma Tese de Doutoramento ecentemente concluída e entregue para admissão a provas públicas de defesa, financiada pela Universidade de Lisboa ao abrigo do Programa de Bolsas de Doutoramento UL (BD2015). 
mundo encontra a sustentação disciplinar (e portanto teorética, epistemológica e metodológica) mais adequada ao seu desenvolvimento enquanto campo de indagação significativo no domínio dos estudos literários.

Palavras-chave: Literatura Comparada; literatura-mundo; enquadramento disciplinar; cosmopolitismo.

ABSTRACT: Despite the recent creation of specialized scientific journals in the field (such as the Journal of World Literature), the occurrence of congresses and cultural events dedicated to it (such as the Festival de Literatura-Mundo do Sal), the creation of institutions that bring together and represent its practitioners (such as the Institute for World Literature, at the University of Harvard) and even its insertion in the scope of university curricula (especially in the US, where they nevertheless tend to assume the configuration of survey courses mostly dedicated to the listing and brief presentation of literary texts "of the world»), I believe it is within the framework of Comparative Literature that world literature finds the disciplinary (and therefore theoretical, epistemological and methodological) support best suited to its development as a significant field of inquiry in the scope of literary studies.

Keywords: Comparative Literature; World Literature; Disciplinary Framework; Cosmopolitanism.

The epoch of World-literature is at hand, and everyone must strive to hasten its approach.

(Goethe em Damrosch 2014, 19-20)

World literature is now once again not only an essential, but a highly innovative aspect of comparative literature's disciplinary history.

(Bermann em D'haen, Damrosch e Kadir 2012, 178)

Every now and then, in the field of comparative literature you get someone making an imperialist claim for their particular thing and saying that the discipline has to become this, for example that comp lit has to be replaced by translation studies ${ }^{2}$. And translation

2. Cf. e.g. BASSNETT $(1993,161)$ : "Comparative literature as a discipline has had its day. Cross-cultural work in women's studies, in post-colonial theory, in cultural studies has changed the face of literary studies generally. We should look upon translation studies as 
studies have come in and they're very important, but this would not be the whole of comp lit. I wouldn't want to be seeming to say "comp lit is now world literature". I think rather that we have a dynamic interplay in which world literature can help reframe comparative study.

(Damrosch em Spivak e Damrosch 2011, 481)

Desde a sua institucionalização académica no final do século XIX, a Literatura Comparada tem vindo a desenvolver-se, enquanto disciplina, pela renovação periódica das suas áreas de investigação mais tradicionalmente estabelecidas (como os estudos temáticos, genológicos, de recepção ou de literatura e história), assim como por via de uma interacção progressiva com outros campos do saber mais recentemente constituídos (como os estudos de género, de tradução, pós-coloniais, interartes ou interculturais, como são os Este-Oeste) e com novas problemáticas culturais e sociais atinentes à realidade contemporânea (como serão hoje, por exemplo, as questões de espaço e cartografia ou as de urbanidade e cidadania) ${ }^{3}$. Digo interacção e não integração, uma vez que o tipo de relacionamento que no âmbito dos estudos de Literatura Comparada se estabelece com outros campos do saber e da cultura não passa, de facto, pela dissolução de uns na outra, ou vice-versa. Tampouco implica, como bem sublinha Damrosch no excerto supra, a substituição de uma pelos outros. Trata-se, na verdade, de um tipo de «interacção dinâmica» da qual resulta, não só a "reformulação dos estudos comparatistas», ou apenas a dos domínios que com estes se relacionam, mas antes uma possibilidade de reconfiguração recíproca. Assim acontece, nomeadamente, com uma das áreas de investigação que, nas últimas duas décadas, maior preponderância tem assumido no âmbito dos estudos de Literatura Comparada: a da literatura-mundo.

Em rigor, o surgimento do conceito de Weltliteratur terá, não apenas precedido historicamente o da Literatura Comparada, como aliás contribuído para a formação da disciplina com a qual, hoje, tão proficuamente

the principal discipline from now on, with comparative literature as a valued but subsidiary subject area».

3. Para uma descrição evolutiva da disciplina de Literatura Comparada, cf. e.g. GuILLÉN ([1985] 2005, 11-24; «La Literatura Comparada y la crisis de las humanidades»), BuesCu (2001, 1-64; «Interrogações e fundamentos do comparatismo»), Brunel e CHEVREL ([1989] 2004, 1-20), Bassnett (1993, 1-47) ou Damrosch, Melas e Buthelezi (2009). 
se inter-relaciona ${ }^{4}$. A ambos subjaz, afinal, «a [...] vision [...] of the world's literary production transcending borders and languages but not in any way effacing their individuality and historical concreteness» (Saïd em Auerbach [1953] 2003, xvi). Não obstante, a disciplina de Literatura Comparada acabaria por se sistematizar, sobretudo até meados do século Xx, adentro de uma tradição francesa que privilegiava os rapports de fait (i.e., as relações literárias concretas e explícitas, com particular destaque para os estudos de influência) entre dois textos literários de proveniência europeia, ou ainda de uma tradição norte-americana no seio da qual a leitura dos textos na sua língua original era indispensável, assim excluindo automaticamente uma larga porção da produção literária mundial do âmbito de uma disciplina que se afirmava no quadro do mundo académico ocidental. A Weltliteratur ocuparia, portanto, uma posição marginal no âmbito dos estudos de Literatura Comparada sensivelmente até aos anos 70, quando então a disciplina entra na sua "fase teórica" ${ }^{5}$ e estreita relações com os campos do saber anteriormente mencionados, desse modo superando boa parte dos constrangimentos linguísticos (sobretudo interagindo com os estudos de tradução) e metodológicos (por via dos estudos culturais e pós-coloniais) que inviabilizavam a expansão do seu escopo de análise a uma escala planetária. E é nessa altura, quando a produção literária mundial passa a ser vista como «um grande espaço/laboratório de traduções, transposições, transmutações, transcriações e empréstimos» (Barrento 2002, 73), que estão pois criadas as condições necessárias para uma activação do conceito de literatura-mundo, inspirado pelo de Weltliteratur mas progressivamente distinto deste.

Por razões também históricas e sociais, para além das epistemológicas, esta activação coincide ainda com a retoma de um pensamento cosmopolita com vista à projecção de novos modelos morais, políticos e culturais de escopo planetário, capazes de dar resposta às diversas mutações geradas no mundo contemporâneo sobretudo a partir dos anos $90^{6}$. Ora,

4. Cf. e.g. Sandra Bermann em D’haen, Damrosch e Kadir (2012, 169-179) ou Steiner ([1996] 2003, 150-166).

5. Não por acaso, encontramos um índice dividido nas secções "Origins", "The years of crisis", "The theory years» e "Contemporary explorations" em Damrosch, Melas e BuTHELEZI (2009).

6. Refiro-me à crescente circulação internacional de bens, tecnologia, informação, imagens e pessoas (a que hoje nos referimos como globalização) e à criação de redes de comunicação global como a Internet (que contribuiriam gradualmente para uma percepção global do mundo como estando mais interconectado, mais interdependente e, nesse sentido, mais unificado do que nunca), assim como à dissolução da União Soviética, em 1991, após a qual estariam reunidas as condições necessárias para que o cosmopolitismo pudesse voltar a ser considerado como hipótese politicamente viável. 
se atendermos ao modo como, por via do cosmopolitismo, 1) se reconhecem e incorporam diferenças (por oposição à tendência para esbatê-las subjacente a uma abordagem universalista), 2) que não são vistas como decorrendo simplesmente do seu enquadramento nacional (diferentemente do que sucede com o internacionalismo) e 3) com as quais, de facto, se interage e negoceiam pertenças (contrariamente ao modelo isolacionista em que acaba por resultar o multiculturalismo), parecem-me evidentes as vantagens de uma reflexão que favoreça (ou, melhor dizendo, reforce ${ }^{7}$ a sua articulação com a noção de literatura-mundo e com a disciplina de Literatura Comparada.

Conduzir uma reflexão no âmbito da literatura-mundo ou da Literatura Comparada que de facto privilegie a sua articulação com o conceito de cosmopolitismo contribuirá, antes de mais, para o desenvolvimento de uma percepção do mundo contemporâneo que o reconheça como sendo composto por culturas que não são auto-contidas, que se cruzam e interrelacionam mediante formas variadas de circulação e de comunicação multilateral (como serão aquelas geradas pelo comércio, pelas viagens e migrações, pelo exílio ou pela diáspora, entre outras) e que simultaneamente reflectem e projectam um certo "processo de recalibração da pólis» (Tihanov em Adams e Tihanov 2011, 134, tradução e destaque meus) correspondente, na verdade, à recalibração do próprio mundo ${ }^{8}$. Do desenvolvimento dessa cosmovisão decorrerá, por sua vez, uma capacidade (ou pelo menos uma possibilidade) de 1) sinalização dos modos pelos quais esse quadro de reciprocidades culturais se materializa nos textos que dele emanam, e de 2) reconhecimento desses mesmos textos como constituindo, aliás, lugares de

7. Uma vez que «o "élan" comparatista específico começa a formular-se, no século XVIII, em torno de uma situação cultural (e até política) que integra dois movimentos específicos e até correlatos: por um lado, o impulso cosmopolitista e inter-nacionalista a que o Iluminismo oferece um suporte filosófico e ideológico; por outro lado, a prática sócio-cultural, que cada vez mais repousa sobre a ideia-chave e o conceito de "nação". Da convergência e mesmo do cruzamento entre estes dois movimentos, na aparência de sinal contrário, nascerá, a pouco e pouco, a proposta de uma disciplina que, nos estudos literários [...], represente a formalização e sistematização de uma metodologia comparativa» (BUESCU, Helena Carvalhão. s.v. "Literatura Comparada". Em E-Dicionário de Termos Literários (EDTL), coord. de Carlos Ceia, último acesso: 07/2019, destaque a negrito meu).

8. "I take cosmopolitanism to be a discursive practice which has to be construed and interpreted historically as a marker that traces the processes of communities (not just the demos of the nation state, but also sub-national or trans-nationally constituted communities) altering their shape and boundaries -and as a result of this also their self-perception- in either direction, expanding towards more inclusive entities or shrinking towards more exclusionist bodies» (Tihanov em ADAMS e TiHANOv 2011, 134, destaques a negrito meus). 
cruzamento e representação nesse sentido privilegiados. E se o cosmopolitismo corresponde ainda, como crê por exemplo Robert Fine, a um gesto e a uma via "to welcome encounters with other cultures as opening a road towards critical self-examination» (em Adams e Tihanov 2011, 155), pareceme ainda significativamente similar o seu funcionamento ao do próprio conceito de literatura-mundo e ainda ao da disciplina de Literatura Comparada. Com efeito, fazem parte de ambos uma dimensão e uma prática auto-reflexivas, decorrentes justamente do seu relacionamento com as várias tradições literárias e culturais com que se «encontram", e que incidem, não apenas sobre os seus objectos de análise, mas também sobre os seus próprios campos cognitivos ${ }^{9}$, sobre os seus limites e sobre as suas potencialidades enquanto áreas conceptuais ou disciplinares, respectivamente. Sendo esta, aliás, uma das características que partilham e uma das razões que justificará, a meu ver, a pertinência dos estudos de literatura-mundo no âmbito disciplinar da Literatura Comparada ${ }^{10}$, onde creio que aqueles encontrem, actualmente, a fundamentação epistemológica de que necessitam para se instituírem como campo de indagação significativo no domínio dos estudos literários.

Nesse sentido, afirma Helena Carvalhão Buescu (2013, 56; 66):

A literatura-mundo pode assim ser compreendida como experiência simultânea do comum e do incomum: arquivo de semelhanças potenciais mas também de diferenças e infinitas variações. Para tal, não pode prescindir de uma perspectiva comparatista. [...] Enquanto o debate norte-americano sempre sublinhou a não-coincidência entre literatura comparada e literatura-mundo [...], penso que a colocação europeia do debate só terá a ganhar com a defesa de que a literatura-mundo não pode prescindir da

9. "Nesse sentido, a "crise" dos estudos comparativos talvez possa deixar de ser vista como um "sintoma" circunstancial, para passar a ser encarada como uma forma de autoreflexão, em sintonia com aquilo a que $\operatorname{KOMAR}(1995,291)$ se refere como modo de "intellectual adjustment", ou seja, uma específica auto-interrogação constitutiva, que GuILLÉN (1985) designava como "inquietação". Tal atitude "inquieta" é, a meu ver, um posicionamento básico a qualquer comparatista, na medida em que o faz interrogar e constituir objectos cujo carácter "dado" nunca pode ser tomado como tal. Mas também na medida em que o comparatista se interroga, com a mesma constância e persistência, sobre o "não-dado" do seu próprio campo e da sua própria posição teórico-crítica - e é justamente nesse sentido que o alcance do procedimento comparativo é, basilarmente, epistemológico» (BUESCU 2001, 35). Sobre a "crise» a que se refere a autora, veja-se Wellek em Damrosch, MELAS e Buthelezi (2009, 161-172).

10. Para René Étiemble, por exemplo, «if comparative literature [...] can be considered in relation to world literature, this is not because it is identical with the latter, but only in so far as it allows us to gain access to it” (em Damrosch 2014, 87, destaque meu). 
epistemologia comparatista, afinal inerente à diversidade da constituição das literaturas europeias e da variabilidade do seu relacionamento com outras não-europeias. É este nomeadamente o caso de Portugal, que não pode imaginar-se como isolado dos contextos geográfico e político pelo qual tanto a Europa como os países lusófonos fazem parte da sua própria imagem e da sua própria história.

Precisamente, julgo que qualquer conceito, ideia ou teoria dependerá sempre do olhar por que for visto, e portanto de um posicionamento teorético mas também cultural sempre localizado ${ }^{11}$. Partilhando ambos com a autora, e por isso concordando em absoluto com as considerações supra, creio ainda que haja pelo menos uma outra razão forte pela qual seja possível justificar a pertinência dos estudos de literatura-mundo no âmbito disciplinar da Literatura Comparada: nomeadamente, a ancoragem textual a que esta sempre obriga. Se é possível equacionar diversas formas de pensar e trabalhar sobre a literatura-mundo, que passarão pela sua interacção com as áreas da sociologia, da economia, da biologia, da cartografia e da tradução, entre outras ${ }^{12}$, penso no entanto que seja priorizando uma aproximação efectiva aos textos que constituem, afinal, o seu objecto central de análise, que a literatura-mundo possa de facto instituir-se como «experiência simultânea do comum e do incomum: arquivo de semelhanças potenciais mas também de diferenças e infinitas variações». Porque é no domínio da literatura que nos situamos, e não em qualquer outro sistema-mundo, a experiência do incomum implicada no conceito de literatura-mundo terá necessariamente de privilegiar (ou pelo menos de começar por) uma prática de leitura aproximada (close reading) consentânea com o gesto de "abertura ao Outro" que se propõe ${ }^{13}$. E não era, pois, nestes termos que Brunel e Chevrel se referiam justamente ao «impulso original da actividade comparatista»? ${ }^{14}$

11. «For any given observer, even a genuinely global perspective remains a perspective from somewhere, and global patterns of the circulation of world literature take shape in their local manifestations" (DAMROSCH 2003, 27).

12. Cf. e.g. Thomas Beebee (em D'haen, Damrosch e Kadir 2012, 297-306), Ann Steiner (em D'haen, Damrosch e Kadir 2012, 316-324) ou Ursula K. Heise (em D'HAEN, DAMROSCH E KADIR 2012, 404-412).

13. Cf. e.g. PatTel (2015) ou Gallop (2000); para Jane Gallop, apenas a leitura aproximada (close reading) do que de facto está escrito permite ver o Outro sem sobre ele projectar os nossos pré-conceitos culturais.

14. "O impulso original da actividade comparatista, a sua razão de ser, a sua metodologia: a abertura ao "outro", àquele que não escreve como nós, que não pensa como nós - que é ele mesmo, na sua diferença e originalidade» (BRunel e CHEVREL [1989] 2004, vii). 
Se a literatura-mundo corresponde, portanto, a um determinado "modo de lers (Damrosch 2003, 281, tradução e destaque meus), julgo ainda que, por meio da sua já referida articulação com o cosmopolitismo (também este uma forma de «abertura ao Outro» $)^{15}$, ela possa igualmente corresponder a um certo modo de fazer literatura. Vejamos, nesse sentido, o trecho seguinte:

Cosmopolitanism is primarily about viewing oneself as part of a world, a circle of belonging that transcends the limited ties of kinship and country to embrace the whole of humanity. However, since one cannot see the universe, the world, or humanity, the cosmopolitan optic is not one of perceptual experience but of the imagination. World literature is an important aspect of cosmopolitanism because it is a type of world-making activity that enables us to imagine a world (Cheah em Delanty 2012, 138).

Se Cheah se refere, no artigo de onde transcrevo o excerto supra ${ }^{16}$, essencialmente ao modo como a literatura-mundo permite re-ler e re-criar $o$ mundo entendido enquanto macro-sistema literário, imaginando-o mais cosmopolita e menos hierarquizado ${ }^{17}$, penso que haja pelo menos uma outra via pela qual a literatura-mundo possa corresponder a um certo «type of world-making activity», e que também Cheah contempla num seu outro estudo posterior: refiro-me à possibilidade de criação de um mundo textual capaz de re-ler e re-criar, em si mesmo e pelas ideias, diálogos e princípios que veicula ou estabelece, $o$ mundo empírico que integra, desse modo instituindo-se como caso de literatura-mundo ${ }^{18}$. Abarcando múltiplos tempos,

15. "Historically, the idea of belonging to, and behaving as the member of, a polis that coincides with the entire world (cosmos) is the earliest discursive articulation of cosmopolitanism, beginning with the Cynics and then the Stoics (with emphasis on cosmopolitanism understood as personal ethos), and later metamorphosing into various discourses - many of them still with us today - promoting heightened awareness and acceptance of cultural difference, primarily by the individual but also, by extension and at a later stage, by the collective» (TiHanov 2011, 134, destaque meu).

16. "What Is a World? On World Literature as World-Making Activity» (em DeLANTY 2012, 138-149).

17. À semelhança, aliás, do comparatismo, também ele destacado como «lugar de uma possível relativização de uma hierarquia pré-determinada” (BuEscu 2001, 7).

18. Em What Is a World?: On Postcolonial Literature as World Literature, CHEAH (2016, 3) procura examinar "World literature's impact qua literature on the world" esboçando "an account of world literature that does not merely describe and analyze how literary works circulate around the world or are produced with a global market in mind but that seeks to understand the normative force that literature can exert in the world, the ethicopolitical horizon it opens up for the existing world» (СНЕAH 2016, 5, destaque meu). 
espaços e vozes (literárias mas não só) que entre si dialogam, e portanto redesenhando possíveis redes de interacção e/ou sugerindo a configuração de novos modelos morais, políticos e culturais de escopo planetário (aludo portanto à materialização de uma certa imaginação cosmopolita), o texto que assim se institui como um mundo e como caso de literatura-mundo poderá, inclusivamente, ter efeitos sobre o mundo do qual emana e para que se projecta, eventualmente, como modelo. Nesse sentido, afirma aliás Birgit Neumann (2018, 253, destaques meus): "Literature affects the world it inhabits by literary acts of worldmaking [and a] comparative history that takes seriously the agency of literary worldmaking deduces categories for comparison from the literary text itself rather than imposing pre-given paradigms from without».

Trata-se, pois, da possibilidade de pensar o texto como um mundo mas, sobretudo, de pensar a literatura como um mundo ${ }^{19}$. Também por isso opto por uma designação hifenizada da literatura-mundo, tomando por base o modo como Wallerstein escolheu grafar a sua teoria dos "sistemas-mundo", e para quem "putting in the hyphen was intended to underline that we are talking not about systems, economies, empires of the (whole) world, but about systems, economies, empires that are a world (but quite possibly, and indeed usually, not encompassing the entire globe)» (Wallerstein 2004, 16-17). Baseio-me ainda, neste e noutros aspectos relativos ao estudo e à teorização da literatura-mundo nos quais este artigo se revê, no modo como Buescu adapta a expressão littérature-monde, proposta em 2007 por um grupo de escritores e intelectuais franceses num manifesto publicado no Le monde des livres $^{20}$, para o seu uso do conceito e da expressão de literatura-mundo ${ }^{21}$. À semelhança da autora, opto ainda por uma grafia do conceito em minúsculas, justamente para distingui-lo da Literatura Comparada que, em maiúsculas, designa com propriedade uma área disciplinar. À literatura-mundo tenho vindo a referir-me utilizando termos como "conceito", "noção" ou "área de investigação», por entender que a mesma não se institua, pelo menos por enquanto, como disciplina autónoma no que isso implica do ponto de vista da produção de instâncias teóricas e metodológicas consensualmente aceites e partilhadas pelos diversos estudiosos que sobre ela se debruçam.

19. «At stake are not the modalities of analyzing literature on a world scale, but the conceptual means for thinking literature as a world" (Casanova em DAmrosch 2014, 194).

20. Disponível em linha em: https://www.lemonde.fr/livres/article/2007/03/15/desecrivains-plaident-pour-un-roman-en-francais-ouvert-sur-le-monde_883572_3260.html (último acesso: 07/2019).

21. Cf. Buescu $(2013,38-39$ e 54-55). 
Pese embora a recente criação de revistas científicas especializadas no campo (como o Journal of World Literature) ${ }^{22}$, a ocorrência de congressos e eventos culturais que lhe são dedicados (como o Festival de LiteraturaMundo do Sal) ${ }^{23}$, a criação de instituições que reúnem e representam os seus praticantes (como o Institute for World Literature, na Universidade de Harvard $)^{24}$ e até mesmo a sua inserção no âmbito dos curricula universitários (sobretudo os norte-americanos, onde não obstante assumem tendencialmente a configuração de survey courses consagrados apenas à listagem e breve apresentação de textos literários «do mundo»), creio de facto que seja, reitero, no âmbito da Literatura Comparada que a literatura-mundo encontra a sustentação disciplinar (e portanto teorética, epistemológica e metodológica) mais adequada ao seu desenvolvimento enquanto área de estudos "concerned with patterns of connection in literatures across both time and space» (Bassnett 1998, 1) ${ }^{25}$. Justamente, creio que seja este seu enquadramento disciplinar o garante de que a literatura-mundo não se esvazia, nem esvaziará, numa área de estudos "culturally deracinated, philologically bankrupt, and ideologically complicit with the worst tendencies of global capitalism» (Spivak em Spivak e Damrosch 2011, 456).

\section{REFERÊNCIAS}

Adams, David e Galin Tihanov, eds. 2011. Enlightenment Cosmopolitanism. Londres: Legenda.

AuERBACH, Erich. (1953) 2003. Mimesis: The Representation of Reality in Western Literature. Traduzido por Willard R. Trask. Princeton/Oxford: Princeton University Press.

Barrento, João. 2002. O Poço de Babel: Para uma poética da tradução literária. Lisboa: Relógio D’Água.

BAssnetT, Susan. 1993. Comparative Literature: A Critical Introduction. Oxford: Blackwell.

Brunel, Pierre e Yves Chevrel, eds. (1989) 2004. Compêndio de Literatura Comparada. Traduzido por Maria do Rosário Monteiro. Lisboa: Fundação Calouste Gulbenkian.

22. Cf. https://brill.com/view/journals/jwl/jwl-overview.xml (último acesso: 07/2019).

23. Cf. https://www.facebook.com/festivaldeliteraturamundosal/(último acesso: 07/2019).

24. Cf. https://iwl.fas.harvard.edu/ (ultimo acesso: 07/2019).

25. Et pour cause, é nestes termos que Bassnett se refere à disciplina de Literatura Comparada. 
Buescu, Helena Carvalhão. 2001. Grande Angular: Comparatismo e práticas de comparação. Lisboa: Fundação Calouste Gulbenkian.

Buescu, Helena Carvalhão. 2013. Experiência do Incomum e Boa Vizinhança. Literatura Comparada e Literatura-Mundo. Porto: Porto Editora.

CHEAH, Pheng. 2016. What Is a World? On Postcolonial Literature as World Literature. Durham/Londres: Duke University Press.

Damrosch, David. 2003. What is World Literature? Princeton: Princeton University Press.

Damrosch, David, ed. 2014. World Literature in Theory. Oxford: Wiley-Blackwell.

Damrosch, David, Natalie Melas e Mbongiseni Buthelezi, eds. 2009. The Princeton Sourcebook in Comparative Literature: From the European Enlightenment to the Global Present. Princeton: Princeton University Press.

Delanty, Gerard, ed. 2012. Routledge Handbook of Cosmopolitan Studies. Nova York: Routledge.

D'haen, Theo, David Damrosch e Djelal Kadir, eds. 2012. The Routledge Companion to World Literature. Nova York: Routledge.

GALLOP, Jane. 2000. "The Ethics of Reading: Close Encounters». Journal of Curriculum Theorizing 16: 7-17.

Guillén, Claudio. (1985) 2005. Entre lo uno y lo diverso. Introducción a la Literatura Comparada (Ayer y boy). 2. ${ }^{\mathrm{a}}$ ed. revisada e aumentada. Barcelona: Tusquets.

NeumanN, Birgit. 2018. "Vernacular Cosmopolitanism in Anglophone World Literatures: Comparative Histories of Literary Worlding». Arcadia: International Journal of Literary Culture / Internationale Zeitschrift für literarische Kultur 53 (2): 239-257.

PATEll, Cyrus R. K. 2015. Cosmopolitanism and the Literary Imagination. Nova York: Palgrave Macmillan.

SPIVAK, Gayatri Chakravorty e David DAmRosCH. 2011. "Comparative Literature/ World Literature». Comparative Literature Studies 48 (4): 455-485.

WallersteIn, Immanuel. 2004. World-Systems Analysis: An Introduction. Durham/ Londres: Duke University Press. 
